\title{
Heterogeneity response to afatinib in gastric cancer patient with uncommon epidermal growth factor receptor (EGFR) mutations: a case report
}

\author{
Qin Liu ${ }^{1}$, Yang Yang ${ }^{1}$, Xiangshan Fan ${ }^{2}$, Xiaoyan Xin ${ }^{3}$, Qiuyue Pan ${ }^{4}$, Yihong Zhang ${ }^{4}$, Baorui Liu ${ }^{1}$, Jia Wei ${ }^{1}$ \\ ${ }^{1}$ The Comprehensive Cancer Centre of Drum Tower Hospital, Medical School of Nanjing University \& Clinical Cancer Institute of Nanjing \\ University, Nanjing, China; ${ }^{2}$ Department of Pathology, Affiliated Drum Tower Hospital of Nanjing University Medical School, Nanjing, China; \\ ${ }^{3}$ Department of Radiology, Affiliated Drum Tower Hospital of Nanjing University Medical School, Nanjing, China; ${ }^{4}$ OrigiMed, Shanghai, China \\ Correspondence to: Jia Wei, MD, PhD. The Comprehensive Cancer Centre of Drum Tower Hospital, Medical School of Nanjing University \& \\ Clinical Cancer Institute of Nanjing University, Nanjing 210008, China. Email: Weijia01627@hotmail.com.
}

\begin{abstract}
Despite concerted efforts that have been made to characterize and understand the genomic landscape of gastric cancer (GC), only HER2 has been validated as a molecular target for GC treatment. Identifying new valid therapeutic targets is important for the treatment of this disease. The present report describes a Chinese male with a history of smoking two packs per day, who did not have a family history of cancer or other hereditary diseases, we discovered a small painless lump in the right groin in February 2018. Histopathology revealed a primary gastric adenocarcinoma. Positron emission tomography-computed tomography (PET-CT) showed multiple hypermetabolic nodules in the right upper lung, greater curvature of the stomach, and muscles. The patient had received treatment included oxaliplatin, docetaxel, and tegafur for two cycles, and second-line therapy of irinotecan and capecitabine, inguinal mass excision followed by concurrent radio-chemotherapy. However, the disease rapidly progressed. Whole exome sequencing (WES) showed uncommon epidermal growth factor receptor (EGFR) mutation of G719S + L861Q. The following EGFR tyrosine kinase inhibitors (TKIs) afatinib demonstrated partial response. Two months after targeted therapy, gastroscopy indicated rapid progression. With subsequent gastric specimen WES analysis, secondary MET amplification was found. The patient received local radiotherapy for gastric lesions as well as oral administration of apatinib. However, the disease rapidly progressed. A month later, he died of hepatic encephalopathy caused by obstructive jaundice combined with pulmonary and biliary tract infection. The present study indicated that afatinib might be a beneficial therapeutic option for a subset of GC patients with rare EGFR mutation in their tumors.
\end{abstract}

Keywords: Case report; gastric cancer (GC); epidermal growth factor receptor mutation (EGFR mutation); afatinib; MET amplification

Submitted Nov 05, 2020. Accepted for publication Apr 02, 2021.

doi: $10.21037 /$ atm-20-7312

View this article at: http://dx.doi.org/10.21037/atm-20-7312

\section{Introduction}

Gastric cancer (GC) is the third leading cause of cancer death worldwide (1). GC based on driver gene mutation detection may benefit patients by facilitating molecular targeted drug development and improving survival in GC patients. The epidermal growth factor receptor (EGFR) was first proposed as a potential anticancer target by Mendelsohn and colleagues in 1988 (2). Overexpression of EGFR has been reported in $24-27 \%$ of all gastric adenocarcinomas and is associated with afatinib sensitivity (3). Despite the fact that anti-EGFR monoclonal antibodies have been widely used in colorectal cancer, the impact of somatic mutations of EGFR on the efficacy of ErbB family inhibitors are not clearly demonstrated in GC. 


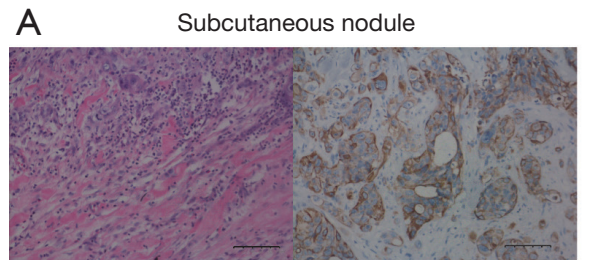

(a) H\&E

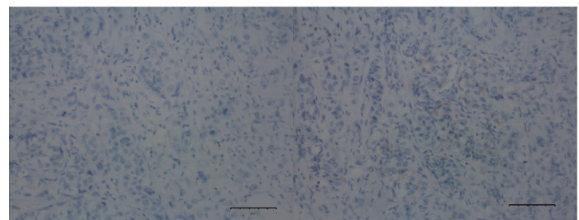

(c) IHC: TTF-1

(d) IHC: VILLIN

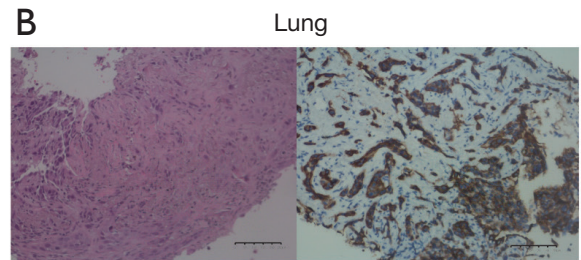

(a) H\&E

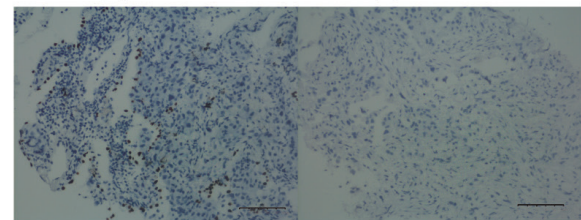

(c) IHC: TTF-1

(d) IHC: VILLIN

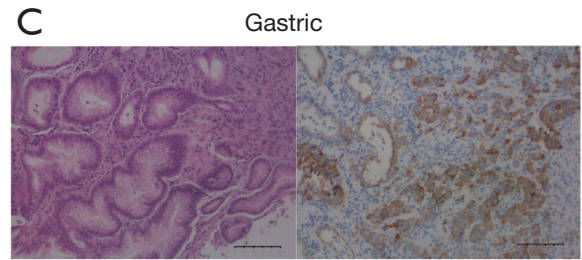

(a) $\mathrm{H} \& \mathrm{E}$

(b) IHC: CK7

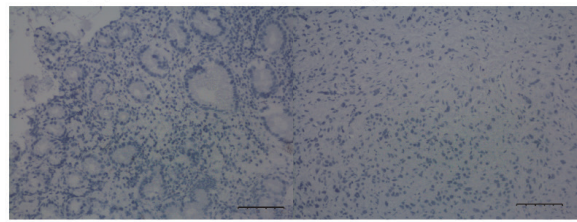

(c) IHC: HER2

(d) IHC: VILLIN

Figure 1 Pathological micrographs and immunohistochemistry of gastric, lung, and subcutaneous nodule. (A) Histological appearance of gastric (hematoxylin and eosin stain, magnification 100x; a), and immunohistochemistry of gastric (magnification 200×; b: CK7, c: HER2, d: Villin). (B) Histological appearance of lung (hematoxylin and eosin stain, magnification 40x; a), and immunohistochemistry of lung (magnification 200x; b: CK7, c: TTF-1, d: Villin). (C) Histological appearance of subcutaneous nodule (hematoxylin and eosin stain, magnification 100×), and immunohistochemistry of subcutaneous nodule (magnification $200 \times$; b: CK7, c: TTF-1, d: Villin).

EGFR inhibitors, such as cetuximab (https://clinicaltrials. gov/show/NCT00655876), panitumumab (http://www. clinicaltrials.gov/show/NCT00824785) and nimotuzumab (http://www.clinicaltrials.gov/show/NCT03400592) are being clinically investigated for efficacy in gastroesophageal cancers (4). However, EGFR somatic mutation frequency is low in GC. A total of 19 (4.5\%) EGFR somatic mutations were identified in two comprehensive molecular profiling studies $(5,6)$, but none of them was a sensitivity mutation. Herein, we report a rare case of a GC patient with a compound EGFR mutation of G719S + L861Q who responded to afatinib, which inform the new predictive somatic mutations for ErbB family target therapy. The report of the mutation is based on whole exome sequencing, which is a promising technique in companion diagnostics in cancer, which is capable to retrieve the complete mutational profile of all relevant genes in one analysis. We present the following article in accordance with the CARE reporting checklist (available at http://dx.doi.org/10.21037/atm-207312).

\section{Case presentation}

The patient, a Chinese male with a history of smoking two packs per day, who did not have a family history of cancer or other hereditary diseases, discovered a small painless lump in the right groin in February 2018. Excisional biopsy and tissue specimen examination revealed a metastatic poorly differentiated adenocarcinoma (Figure 1A). Based on immunohistochemistry (IHC) analysis, which revealed CK(+++), Vim(+++), S100(-), HMB45(-), CK7(+++), CK20(++), Villin(+), PAX8(-), CDX-2(+), PsAP(-), TTF$1(-)$ and CK5/6(-), it was considered to originate from the digestive tract (Figure 1A). Biopsy specimens of the lung and stomach showed poorly differentiated adenocarcinoma, and IHC analysis showed Her-2(0), CK7(+++), CK20(-), Villin(-), TTF-1(-), CK5/6(++), CDX-2(-), P40(+), p63(+) (Figure 1B,C). All of these results demonstrated a primary gastric adenocarcinoma with multiple distant metastases. Multiple hypermetabolic nodules in the right upper lung, greater curvature of the stomach, and muscles were demonstrated by positron emission tomography-computed tomography (PET-CT) (Figure 2A).

Treatment included oxaliplatin, docetaxel and tegafur for two cycles beginning in March 2018, but, unfortunately, the inguinal mass relapsed (Figure 2B). Though secondline therapy of irinotecan and capecitabine were given, the inguinal mass continued to increase. In May 2018, the patient underwent inguinal mass excision followed by concurrent radio-chemotherapy. The patient experienced progressive aggravation in July 2018, and a PET-CT showed new osseous metastases and multiple nodules in the chest wall, abdominal wall and back (Figure 2C). Based on whole exome sequencing (WES) of the inguinal tissue specimen and matching blood sample, a compound EGFR mutation of G719S (c.2155G>A) + L861Q (c.2582T>A) 
A

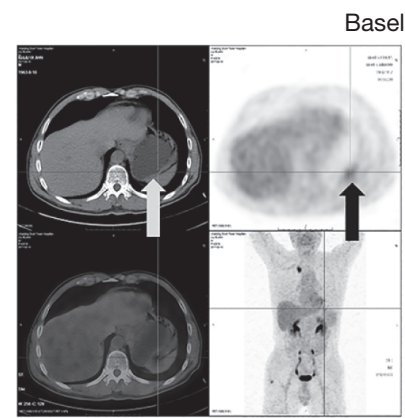

C

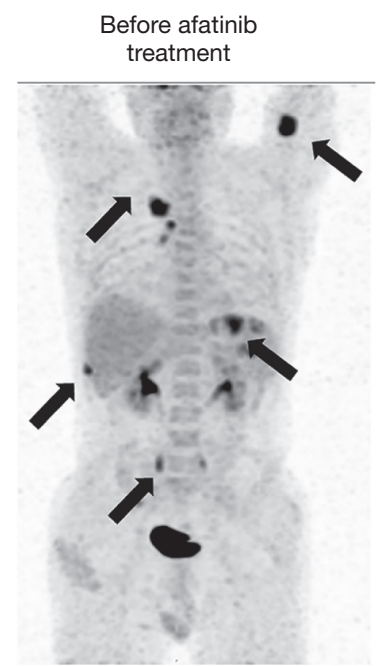

Baseline

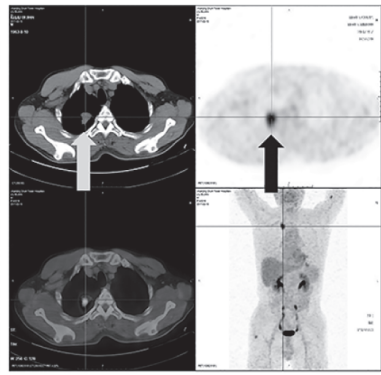

D One month after afatinib treatment

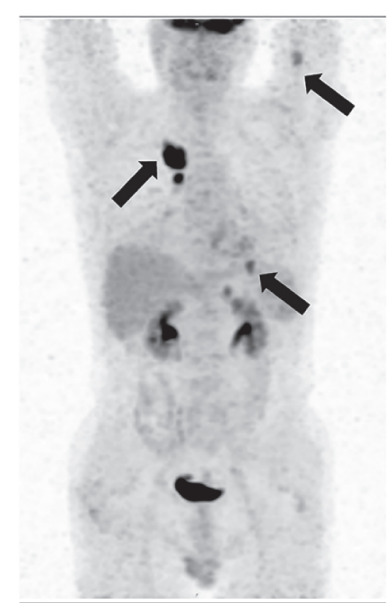

B

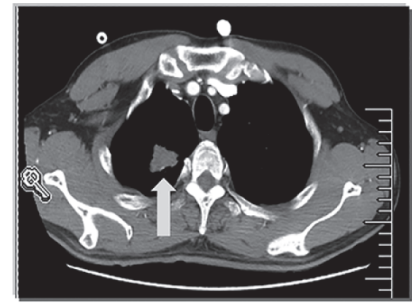

After first-line chemotherapy

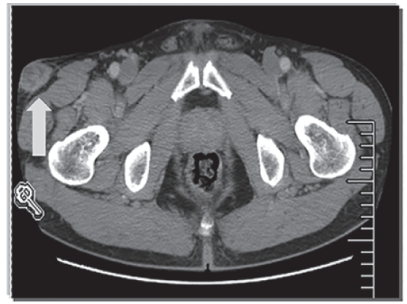

$\mathrm{E}$

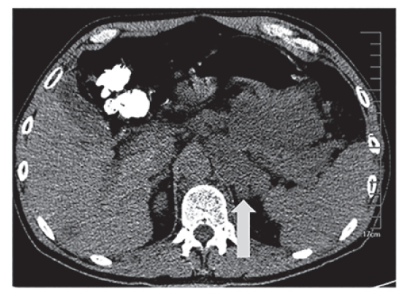

$\mathrm{F}$

Pulmonary infection

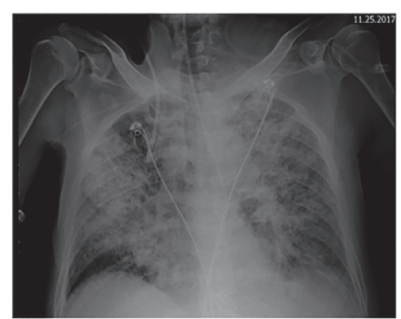

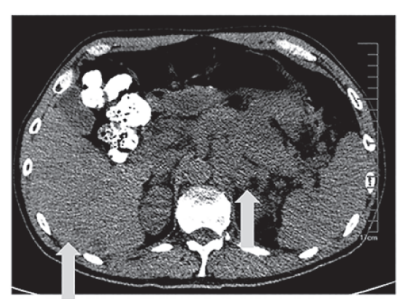

G Two and a half months after afatinib treatment

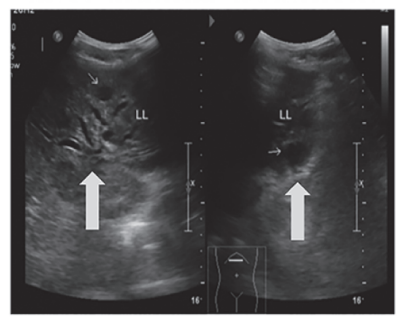

Figure 2 Images after chemotherapy and afatinib. (A) PET-CT showed high metabolic nodules in the greater curvature of the stomach and right upper lung. (B) CT scan after 2 weeks of chemotherapy showed the inguinal mass had relapsed. (C) PET-CT scan after secondline chemotherapy showed newly osseous metastases and multiple nodules in the chest wall, abdominal wall and back. (D) PET-CT scan at 1 month after afatinib treatment showed a significant reduction in size and uptake of the gastric mass, subcutaneous nodules and pelvic metastases, but the right lung tumor had progressed. (E) CT scan 2 months after afatinib treatment showed new multiple liver and left adrenal gland metastases. After two and a half months after afatinib treatment, chest X-ray showed pulmonary infection (F) and ultrasound showed multiple liver metastases as well as bile duct dilatation $(\mathrm{G})$. The arrow points to the location of the lesion, including mass or nodules. PET, positron emission tomography; CT, computed tomography.

and EGFR amplification were detected. The compound EGFR mutation of G719S + L861Q was also found in both the lung and gastric specimens, but EGFR amplification was found only in the inguinal tissue. Gilotrif ${ }^{\circledR}$ (afatinib) was approved for non-resistant EGFR mutations, including L861Q, G719X and S768I. The patient received the treatment of oral afatinib (40 $\mathrm{mg}$, once daily) in August 2018. A month later, the follow-up PET-CT scan showed a significant reduction of the gastric mass, subcutaneous nodules and pelvic metastases, but showed progression of the right lung tumor (Figure 2D). During ongoing treatment, a repeated biopsy of the right lung tumor revealed metastatic poorly differentiated adenocarcinoma, and WES analysis revealed the previously detected compound EGFR mutation of G719S + L861Q. While on afatinib, the patient's physical and psychological health condition improved immensely, as evident by a marked decrease in subcutaneous nodules size and gastric lesions. Two months after targeted therapy, the patient experienced upper abdomen swelling and pain. A gastroscopy indicated rapid progression of the gastric lesion and a follow-up CT scan showed new multiple liver and left adrenal gland metastases (Figure 2E). With subsequent gastric specimen WES analysis, a secondary tyrosine-protein kinase Met (MET) amplification was found, which is postulated to be a potential resistance mechanism in gastric lesion in this 


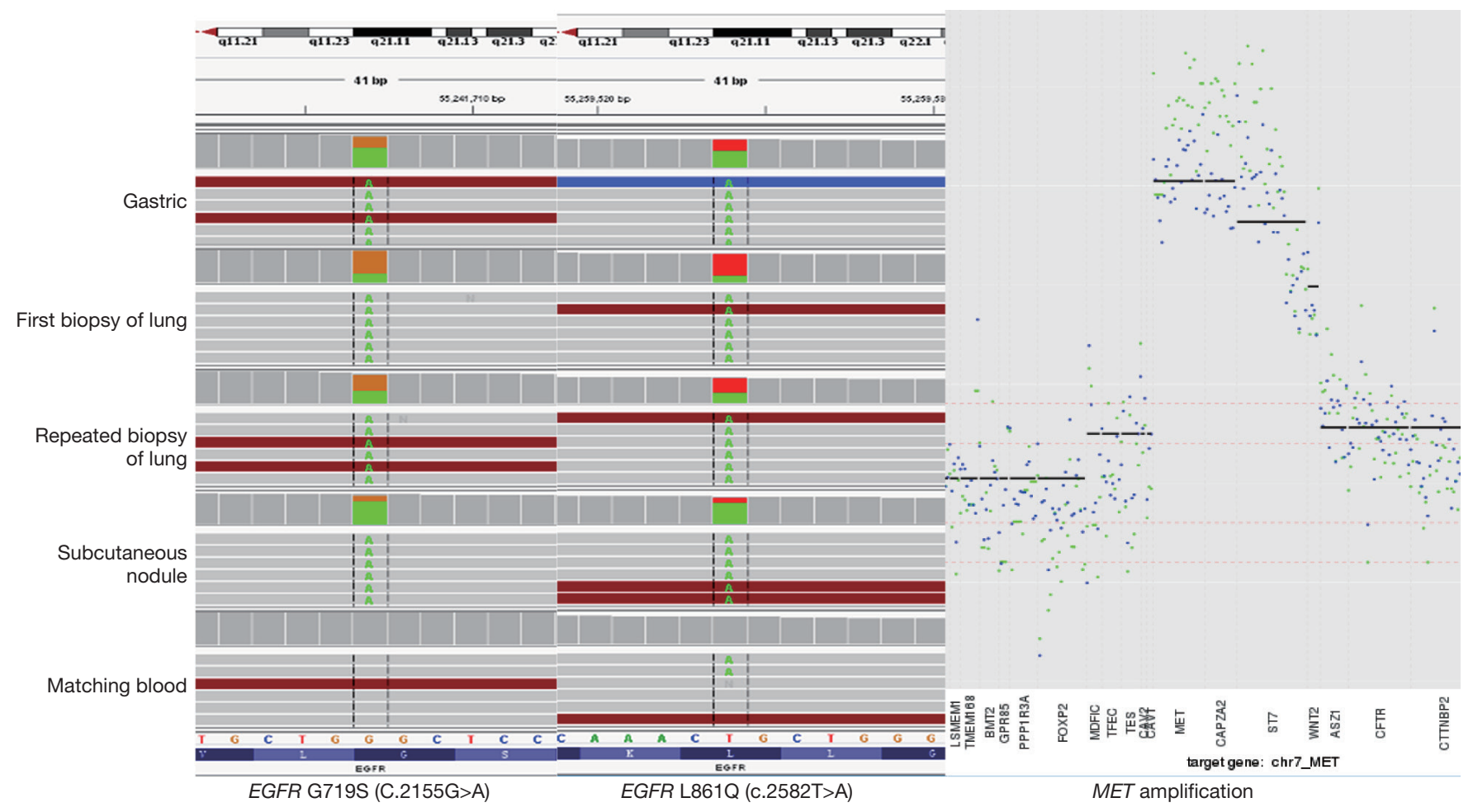

Figure 3 Tumor DNA sequencing at diagnosis and after progression. Sequencing reads from the inguinal tumor, gastric, and lung biopsy at diagnosis detected EGFR G719S (c.2155G>A) + L861Q (c.2582T>A) mutations. Sequencing of a repeat biopsy of the gastric lesion after progression with afatinib treatment demonstrated persistence of the EGFR G719S + L861Q mutation and the acquisition of MET amplification. EGFR, epidermal growth factor receptor.

patient. Afterwards, the patient received local radiotherapy for gastric lesions as well as Oral administration of apatinib. However, the disease rapidly progressed, the chest X-ray showed extensive pulmonary infection and ultrasound scan showed progressive enlargement of multiple liver metastases as well as bile duct dilatation (Figure $2 F, G$ ). Immediately, the patient was cachexic with an Eastern Cooperative Oncology Group (ECOG) score of 4. Therefore, he could not tolerate further treatment. A month later, he died of hepatic encephalopathy caused by obstructive jaundice combined with pulmonary and biliary tract infection. The mutation of EGFR in inguinal tissue specimen, lung and gastric specimens and the tyrosine-protein kinase MET amplification in stomach are shown in Figure 3. This research was approved by the Research Ethics Committee of the Comprehensive Cancer Centre of Drum Tower Hospital (number/ID of the ethics approval: 2016-196), and written informed consent for participate of the clinical details and images was obtained from the patient' relatives.

All procedures performed in studies involving human participants were in accordance with the ethical standards of the institutional and/or national research committee(s) and with the Helsinki Declaration (as revised in 2013).

\section{Discussion}

GC is a highly heterogeneous disease with various histological phenotypes and molecular diversity. Ichikawa et al. (2017) detected at least one alteration in 194 GCs (93.7\%) and 69 actionable genes in 141 GCs (68.1\%) by comprehensive genomic sequencing (7). Here, we are the first to report a rare case of a GC patient responding to afatinib in which a compound EGFR mutation of G719S + L861Q was detected.

G719S and L861Q, generally recognized as sensitizing mutations, with response rates (RRs) and disease control rates (DCRs) comparable to those reported with a mutation of L858R and exon 19 deletions (8). However, the frequency of compound (i.e., double or complex) EGFR mutations and its response/resistance pattern to EGFR tyrosine kinase 
inhibitors (TKIs) are less well described. In a post-hoc analysis, median progression-free survival (PFS) was 13.8 months and median overall survival (OS) was 26.9 months in patients with EGFR G719S mutations who were given afatinib. Patients with EGFR G719S mutations had a substantially longer PFS and OS than those with L861Q (9). A review has summarized that all 12 non-small cell lung cancers (NSCLCs) with a compound EGFR mutation of G719X + L861Q responded to gefitinib or erlotinib, including 10 partial response (PR) and 2 stable disease (SD) (8). In a large retrospective study from multiple Chinese centers, patients harboring complex mutations of G719X + L861Q obtained a RR of $88.9 \%$ and a DCR of $100.0 \%$ after treatment with the first generations of TKIs. These values are significantly higher than those reported for any single mutation (RR 36.8\%, DCR 72.4\% for G719X; RR 39.6\%, DCR 75.5\% for L861Q) $(9,10)$. These outcomes demonstrated that compound EGFR mutations of G719S + L861Q was associated with favorable effectiveness of TKIs.

In this case, EGFR mutation of G719S + L861Q were detected in inguinal, lung and gastric specimens, which means that these mutations were probably trunk mutation. After receiving afatinib, the gastric mass, significant reduction was detected in subcutaneous nodules and pelvic metastases, while not in the lung tumor. The time to control the disease in this GC patient was significantly shorter than that of NSCLC, which may suggest that GC has its organic particularity. Due to the rarity of EGFR sensitive mutation in GC, the mechanism of rapid drug resistance is not clear.

After afatinib treatment, the patient had an initial PR with significant reduction of the gastric lesion and subcutaneous nodules, but the lung metastasis was enlarged. WES results revealed EGFR amplification in gastric and subcutaneous nodules, but not in the lung. The TKI responsiveness was strongly associated with EGFR mutations and only marginally significant in association with increased EGFR copy numbers (11). Evidence for an enhanced effect of TKI induced by EGFR mutations and amplification is limited. Our case was limited in that we did not further investigate the mechanism whereby TKI was effective for gastric lesions but not for lung lesions. The patient developed rapid progression after 2 months of targeted therapy. Re-biopsy of the gastric lesion revealed MET amplification, which is one mechanism of EGFR-TKI resistance and accounts for approximately $5 \%$ of EGFRTKI resistant-related cases (12). MET amplification is one of the most important resistant mechanisms in NSCLC, and MET inhibitors combined with EGFR-TKI can overcome this resistance (13). Whether MET amplification is the cause of resistance to EGFR-TKI in GC remains unknown, due to EGFR sensitive mutation is uncommon alteration.

Sanchez-Vega et al. (2019) find out that the sensitivity response of afatinib was associated with co-amplification of EGFR/ERBB2, but resistance response with amplification of MET in GC patients (14). In this case, the resistance to afatinib in lung metastasis is evident in the secondary amplification of MET, which is consistent to the prior study. Unfortunately, the patient was in poor condition and could not tolerate further treatment. In this case, the lung lesions never responded to afatinib, and we speculate that it is caused by tumor heterogeneity.

We reported a case of advanced GC with a compound EGFR G719S + L861Q mutation. The patient had a transient and mixed response with afatinib. The use of next-generation sequencing in patients with GC may lead, occasionally, to the identification of driver mutations and, as such, may also be targeted by available kinase inhibitors.

\section{Acknowledgments}

Funding: This work was supported by the National Natural Science Foundation of China (No. 81972309) and Jiangsu Provincial Medical Youth Talent (No. QNRC 2016045).

\section{Footnote}

Reporting Checklist: All authors have completed the CARE reporting checklist. Available at http://dx.doi.org/10.21037/ atm-20-7312

Conflicts of Interest: All authors have completed the ICMJE uniform disclosure form (available at http://dx.doi. org/10.21037/atm-20-7312). QP and YZ are employees of OrigiMed. The other authors have no conflicts of interest to declare.

Ethical Statement: The authors are accountable for all aspects of the work in ensuring that questions related to the accuracy or integrity of any part of the work are appropriately investigated and resolved. This research was approved by the Research Ethics Committee of the Comprehensive Cancer Centre of Drum Tower Hospital (number/ID of the ethics approval: 2016-196). All procedures performed in studies involving human participants were in accordance with the ethical standards of the institutional and/or national research committee(s) and 
with the Helsinki Declaration (as revised in 2013). Written informed consent was obtained from the patient' relatives.

Open Access Statement: This is an Open Access article distributed in accordance with the Creative Commons Attribution-NonCommercial-NoDerivs 4.0 International License (CC BY-NC-ND 4.0), which permits the noncommercial replication and distribution of the article with the strict proviso that no changes or edits are made and the original work is properly cited (including links to both the formal publication through the relevant DOI and the license). See: https://creativecommons.org/licenses/by-nc-nd/4.0/.

\section{References}

1. Bray F, Ferlay J, Soerjomataram I, et al. Global cancer statistics 2018: GLOBOCAN estimates of incidence and mortality worldwide for 36 cancers in 185 countries [published correction appears in CA Cancer J Clin. 2020 Jul;70(4):313]. CA Cancer J Clin 2018;68:394-424.

2. Mendelsohn J. Growth factor receptors as targets for antitumor therapy with monoclonal antibodies. Prog Allergy 1988;45:147-60.

3. Park SR, Kook MC, Choi IJ, et al. Predictive factors for the efficacy of cetuximab plus chemotherapy as salvage therapy in metastatic gastric cancer patients. Cancer Chemother Pharmacol 2010;65:579-87.

4. Kim MA, Lee HS, Lee HE, et al. EGFR in gastric carcinomas: prognostic significance of protein overexpression and high gene copy number. Histopathology 2008;52:738-46.

5. Wang K, Yuen ST, Xu J, et al. Whole-genome sequencing and comprehensive molecular profiling identify new driver mutations in gastric cancer. Nat Genet 2014;46:573-82.

6. Cancer Genome Atlas Research Network. Comprehensive molecular characterization of gastric adenocarcinoma. Nature 2014;513:202-9.

Cite this article as: Liu Q, Yang Y, Fan X, Xin X, Pan Q, Zhang Y, Liu B, Wei J. Heterogeneity response to afatinib in gastric cancer patient with uncommon epidermal growth factor receptor (EGFR) mutations: a case report. Ann Transl Med 2021;9(9):814. doi: 10.21037/atm-20-7312
7. Ichikawa H, Nagahashi M, Shimada Y, et al. Actionable gene-based classification toward precision medicine in gastric cancer. Genome Med 2017;9:93.

8. Wu JY, Yu CJ, Chang YC, et al. Effectiveness of tyrosine kinase inhibitors on "uncommon" epidermal growth factor receptor mutations of unknown clinical significance in nonsmall cell lung cancer. Clin Cancer Res 2011;17:3812-21.

9. Yang JC, Sequist LV, Geater SL, et al. Clinical activity of afatinib in patients with advanced non-small-cell lung cancer harbouring uncommon EGFR mutations: a combined post-hoc analysis of LUX-Lung 2, LUX-Lung 3, and LUX-Lung 6. Lancet Oncol 2015;16:830-8.

10. Chiu CH, Yang CT, Shih JY, et al. Epidermal Growth Factor Receptor Tyrosine Kinase Inhibitor Treatment Response in Advanced Lung Adenocarcinomas with G719X/L861Q/S768I Mutations. J Thorac Oncol 2015;10:793-9.

11. Chang JW, Liu HP, Hsieh MH, et al. Increased epidermal growth factor receptor (EGFR) gene copy number is strongly associated with EGFR mutations and adenocarcinoma in non-small cell lung cancers: a chromogenic in situ hybridization study of 182 patients. Lung Cancer 2008;61:328-39.

12. Camidge DR, Pao W, Sequist LV. Acquired resistance to TKIs in solid tumours: learning from lung cancer. Nat Rev Clin Oncol 2014;11:473-81.

13. Deng L, Kiedrowski LA, Ravera E, et al. Response to Dual Crizotinib and Osimertinib Treatment in a Lung Cancer Patient with MET Amplification Detected by Liquid Biopsy Who Acquired Secondary Resistance to EGFR Tyrosine Kinase Inhibition. J Thorac Oncol 2018;13:e169-72.

14. Sanchez-Vega F, Hechtman JF, Castel P, et al. EGFR and MET Amplifications Determine Response to HER2 Inhibition in ERBB2-Amplified Esophagogastric Cancer. Cancer Discov 2019;9:199-209. 